\title{
A Study of Large Plastic Deformations in Dual Phase Steel Using Digital Image Correlation and FE Analysis
}

\author{
V. Tarigopula $\cdot$ O.S. Hopperstad $\cdot$ M. Langseth $\cdot$ \\ A.H. Clausen • F. Hild • O.-G. Lademo $・$ M. Eriksson
}

Published online: 14 February 2008

(C) Society for Experimental Mechanics 2008

The publisher regrets that the following errors appeared in "A Study of Large Plastic Deformations in Dual Phase Steel Using Digital Image Correlation and FE Analysis," by V. Tarigopula et al., in Experimental Mechanics.

An incorrect version of Table 2 was published. Please see below for corrected version.

On the same page as Table 2 in the article, an incorrect symbol was published. $\hat{\sigma}$ should be $\dot{\hat{\sigma}}$. The corrected sentence is as follows: The linear hypoelastic relation, which

The online version of the original article can be found at http://dx.doi. org/10.1007/s11340-007-9066-4.

V. Tarigopula $(\bowtie) \cdot$ O.S. Hopperstad $\cdot$ M. Langseth

A.H. Clausen - O.-G. Lademo

Structural Impact Laboratory (SIMLab),

Centre for Research-based Innovation (CRI),

Department of Structural Engineering,

Norwegian University of Science and Technology,

7491 Trondheim, Norway

e-mail: venkatapathi.tarigopula@ntnu.no

F. Hild

Laboratoire de Mécanique et Technologie (LMT-Cachan),

Ecole Normale Supérieure de Cachan / CNRS-UMR 8535 /

Université Paris 6,

61 Avenue du Président Wilson,

94235 Cachan Cedex, France

O.-G. Lademo $\cdot$ M. Eriksson

SINTEF Materials and Chemistry,

7465 Trondheim, Norway
Table 2 Basic constitutive equations for shell (co-rotational) and brick elements

$\begin{array}{ll}\text { Shell element formulation } & \begin{array}{l}\text { Brick element formulation } \\ \text { (co-rotational) }\end{array}\end{array}$

Additive decomposition of rate-of-deformation tensor into elastic and plastic parts

$\widehat{\mathbf{D}}=\widehat{\mathbf{D}}^{e}+\widehat{\mathbf{D}}^{p}, \quad \widehat{\mathbf{D}}=\mathbf{R}^{T} \cdot \mathbf{D} \cdot \mathbf{R} \quad \mathbf{D}=\mathbf{D}^{e}+\mathbf{D}^{p}$

Hypoelastic stress-strain relation on rate form

$\dot{\hat{\sigma}}=\mathbf{C}: \widehat{\mathbf{D}}^{e}, \quad \widehat{\sigma}=\mathbf{R}^{T} \cdot \sigma \cdot \mathbf{R} \quad \sigma^{\nabla J}=\mathbf{C}: \mathbf{D}^{e}$

Yield criterion

$f(\widehat{\sigma}, R)=\bar{\sigma}(\widehat{\sigma})-\left(\sigma_{0}+R\right) \leq 0 \quad f(\sigma, R)=\bar{\sigma}(\sigma)-\left(\sigma_{0}+R\right) \leq 0$

Associated flow rule

$\widehat{\mathbf{D}}^{p}=\dot{\lambda} \frac{\partial f}{\partial \widehat{\sigma}}, \quad \dot{\bar{\varepsilon}}=-\dot{\lambda} \frac{\partial f}{\partial R}=\dot{\lambda} \quad \mathbf{D}^{p}=\dot{\lambda} \frac{\partial f}{\partial \sigma}, \quad \dot{\bar{\varepsilon}}=-\dot{\lambda} \frac{\partial f}{\partial R}=\dot{\lambda}$

Loading/unloading conditions

$f(\widehat{\sigma}) \leq 0 ; \dot{\lambda} \geq 0 ; f \dot{\lambda}=0 \quad f(\sigma) \leq 0 ; \dot{\lambda} \geq 0 ; f \dot{\lambda}=0$

defines the objective stress rate $\sigma^{\nabla J}$ (or $\dot{\hat{\sigma}}$ ) in terms of elastic rate-of-deformation $\mathbf{D}^{\mathrm{e}}$ (or $\widehat{\mathbf{D}}^{e}$ ), is assumed to be isotropic, i.e. $\mathbf{C}$ is an isotropic fourth order tensor, which is determined by Young's modulus $E$ and Poisson's ratio $i$.

The reference for Wang et al. was omitted from citation list. The full reference is as follows:

Wang T, Hopperstad OS, Lademo OG, Larsen PK (2007) Finite element analysis of welded beam-to-column joints in aluminum allow EN AW 6082 T6. Finite Elem Anal Des 44 (1-2):1-16. 